\title{
Observing conversational laughter in frontotemporal dementia
}

\author{
Peter S Pressman, 1,2,3 Michaela Simpson, ${ }^{3}$ Kelly Gola, ${ }^{2}$ Suzanne M Shdo, ${ }^{2}$ \\ Edoardo G Spinelli, ${ }^{2}$ Bruce L Miller, ${ }^{2}$ Maria Luisa Gorno-Tempini, ${ }^{2}$ Katherine Rankin, ${ }^{2}$ \\ Robert W Levenson ${ }^{3}$
}

\begin{abstract}
'Rocky Mountain Alzheimer's Disease Center, Department of Neurology, University of Colorado, Denver, Colorado, USA

${ }^{2}$ Memory and Aging Center, Department of Neurology, University of California, San Francisco, California, USA ${ }^{3}$ Berkeley Psychophysiology Laboratory, University of California, Berkeley, California, USA
\end{abstract}

\section{Correspondence to} Dr Peter S Pressman, Memory and Aging Center, Department of Neurology, University of California, San Francisco, 675 Nelson Rising Lane, Suite 190, San Francisco, California 94158, USA; ppressman@memory.ucsf. edu, peter.pressman@ucdenver. edu

Received 19 September 2016 Revised 28 December 2016 Accepted 11 January 2017 Published Online First 24 February 2017

\section{ABSTRACT}

Background We performed an observational study of laughter during seminaturalistic conversations between patients with dementia and familial caregivers. Patients were diagnosed with (1) behavioural variant frontotemporal dementia (bvFTD), (2) right temporal variant frontotemporal dementia (rtFTD), (3) semantic variant of primary progressive aphasia (svPPA), (4) non-fluent variant primary progressive aphasia (nfvPPA) or (5) early onset Alzheimer's disease (eoAD). We hypothesised that those with bvFTD would laugh less in response to their own speech than other dementia groups or controls, while those with rtFTD would laugh less regardless of who was speaking.

Methods Patients with bvFTD ( $n=39)$, svPPA $(n=19)$, rtFTD $(n=14)$, nfvPPA $(n=16)$, eoAD $(n=17)$ and healthy controls ( $n=156)$ were recorded (video and audio) while discussing a problem in their relationship with a healthy control companion. Using the audio track only, laughs were identified by trained coders and then further classed by an automated algorithm as occurring during or shortly after the participant's own vocalisation ('self' context) or during or shortly after the partner's vocalisation ('partner' context).

Results Individuals with bvFTD, eoAD or rtFTD laughed less across both contexts of self and partner than the other groups. Those with bvFTD laughed less relative to their own speech comparedwith healthy controls. Those with nfvPPA laughed more in the partner context compared with healthy controls.

Conclusions Laughter in response to one's own vocalisations or those of a conversational partner may be a clinically useful measure in dementia diagnosis.

\section{INTRODUCTION}

Laughter is fundamentally interpersonal, serving myriad social purposes. We are roughly 30 times more likely to laugh when with other people than when alone. ${ }^{1}$ Laughter serves to ease the tension when social norms are violated by another person or by oneself. ${ }^{2}$ Consequently, there are two primary contexts for conversational laughter: laughing at oneself and laughing at others. Laughing at oneself requires sufficient self-monitoring and situational monitoring to recognise that one has violated a societal norm, and requires a desire to soften the impact of that violation. The accompanying feeling is often a self-conscious emotion, typically embarrassment. Laughing at others also requires recognition of a violated social norm, but is more focused externally and less focused on the self. The accompanying feeling is often less self-conscious, typically amusement. Because of these differences in information processing and associated emotions, these two kinds of laughter likely rely on different neural networks, which may be impacted to different degrees by different neurodegenerative diseases. ${ }^{3-5}$

The frontotemporal dementias are a heterogeneous group of degenerative disorders often associated with loss of interpersonal ability. The behavioural variant of frontotemporal dementia (bvFTD) is particularly associated with inappropriate social behaviour. ${ }^{6-8}$ People with bvFTD have less capacity for self-conscious emotion such as embarassment. ${ }^{9}$ Right temporal lobe predominant frontotemporal dementia ( $\mathrm{rtFTD}$ ) is a subtype of bvFTD which also leads to diminished interpersonal ability, likely due to deficits in social semantics. ${ }^{10}$ Left temporal predominant semantic variant of primary progressive aphasia (svPPA) presents with deficits in linguistic semantics and is also associated with abnormal social behaviour, but not to the extent of rtFTD. ${ }^{11}{ }^{12}$ In contrast, the non-fluent variant of PPA (nfvPPA) is usually characterised as socially normal, despite difficulty with speech and grammar. ${ }^{13}$ Early onset Alzheimer's disease (eoAD) can mimic any of these diseases, and is often clinically confused with bvFTD. ${ }^{14}$

We investigated spontaneous laughter during seminaturalistic conversations between patients with bvFTD, nfvPPA, rtFTD, svPPA or eoAD and their familial caregivers. Because bvFTD is associated with decreased self-conscious emotion, ${ }^{15}$ we predicted that patients with bvFTD would laugh less in the context of self (defined as occurring during or shortly after their own speech) compared with other patient groups or controls. Based on literature suggesting a loss of semantic knowledge regarding social norms (which likely produces insensitivity to violations by self or by others), ${ }^{16}{ }^{17}$ as well as literature implicating the temporal lobe in recognising humorous situations, ${ }^{4}{ }^{5}$ we predicted that those with rtFTD would laugh less than controls during conversations regardless of context. We did not expect to find differences in laughter between those with svPPA, nfvPPA or eoAD and controls.

\section{METHODS}

\section{Study design}

Participants with dementia were evaluated initially at the Memory and Aging Center of the University 
of California, San Francisco (UCSF). Couples (participants with dementia and a familial caregiver) were evaluated later (mean 34.8 days) at the Berkeley Psychophysiology Laboratory of the University of California, Berkeley (UC Berkeley). All assessments were conducted between 2002 and 2014.

\section{Participants and diagnostic assessment}

Patients with bvFTD $(n=39), \operatorname{svPPA}(n=19), \operatorname{rtFTD}(n=14)$, nfvPPA $(n=16)$ and eoAD $(n=17)$ were recruited at UCSF and studied at UC Berkeley along with a healthy family member $(n=156)$. In order to optimise the sample size of healthy controls, we included healthy family members from conversations with patients diagnosed with disorders not of interest to this study, such as progressive supranuclear palsy and corticobasal degeneration without comorbid dementia symptoms. All study participants provided written consent regarding study participation. The institutional review boards of UCSF and UC Berkeley approved the study.

Patients were initially diagnosed by a panel of behavioural neurologists and neuropsychologists. BvFTD diagnoses were determined using the Neary clinical criteria ${ }^{18}$; svPPA and nfvPPA were diagnosed using consensus criteria. ${ }^{19} \mathrm{AD}$ was diagnosed using the National Institute on Aging-Alzheimer's Association Diagnostic Guidelines, and included patients with various degrees of behavioural, memory, executive, linguistic and visuospatial dysfunction. ${ }^{20}$ Fourteen participants were diagnosed with rtFTD by a panel of neurologists, neuropsychologists and speech and language specialists using available examination and structural MRI data. ${ }^{21}$ Additional data collected on patients included a semistructured history and physical examination and standardised neuropsychological screenings, which included measures of social behaviour, cognition, memory and language function. Demographic data obtained for patients included age, sex, years of education and handedness. Demographic data obtained for controls included sex and age.

\section{Task description}

Laboratory procedures for obtaining samples of conversations were derived from those originally developed by Levenson and Gottman. ${ }^{22}$ Dyads were instructed to discuss a mutually selected topic of continuing disagreement in their relationship. Each conversation lasted between 10 to $15 \mathrm{~min}$. Audio recordings of the conversations were obtained using unidirectional lavalier microphones attached to each participant.

\section{Acoustic labeling}

Audio data from the conflict conversation were saved as WAV files. Speech and non-speech sounds were labelled in Praat V.5.4.01 (2014), an acoustic analysis program. ${ }^{23}$ The speech of each partner was distinguished from non-speech utterances and the other partner's speech by trained research assistants blinded to the speaker's diagnoses. Environmental noise was also labelled for exclusion from analysis (figure 1). Non-lexical vocal communications with a tight lexical association, such as 'mm-hmm' for 'yes,' were included as speech, as were pause fills (eg, 'um', 'ah', 'er', 'uh' and 'eh').

\section{Measures}

Trained coders listened to the recordings of the conversations and labelled speech and laughs based on their own judgment of what constituted laughter. Based on a previously derived procedure, ${ }^{24}$ when a laugh was detected it was further categorised as being in the 'self' context if the laugh occurred during

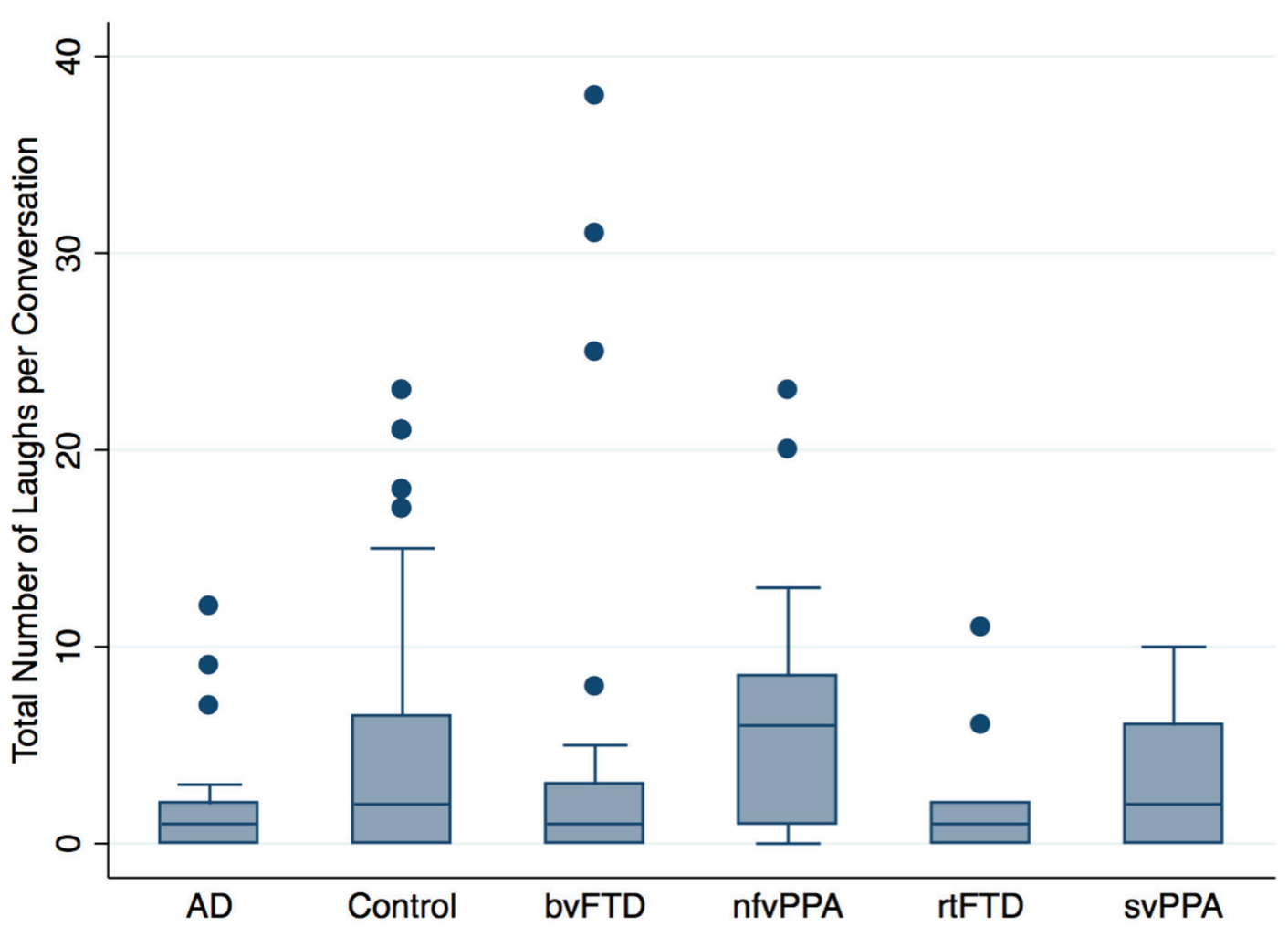

Figure 1 A comparison between different diagnostic groups of total laugh counts per conversation. On the average, participants with AD, bvFTD and rtFTD laughed less often than healthy controls. AD, Alzheimer's disease; bvFTD, behavioural variant frontotemporal dementia; nfvPPA, non-fluent variant primary progressive aphasia; rtFTD, right temporal variant frontotemporal dementia; svPPA, semantic variant of primary progressive aphasia. 
or within $3 \mathrm{~s}$ following that person's own speech, or as occurring in the 'other' context if the laugh occurred during or within $3 \mathrm{~s}$ following the partner's speech or partner's laughter. An additional context, 'spontaneous,' included laughter that occurred $>3 \mathrm{~s}$ after any vocalisation, but was dropped from analysis due to the rarity of occurrence ( $2 \%$ of all laughs). The secondary categorisation of laughs was done automatically using a script written as a Stata 'do-file.' The automated categorisation of the first 100 laughs was compared with a human rater, with 100\% agreement. To control for differences in amount of speech, an automated syllable counter was implemented in Praat for use as a covariate. ${ }^{22}$

\section{Statistical analysis}

Statistical analysis was performed using Stata V.13.0, 2013 (StataCorp; College Station, Texas, USA) and R V.3.1.2, 2014 (R Foundation for Statistical Computing). Independence of laughter per person and per conversation was tested and a mixed effects model was consequently deemed appropriate. A mixed effects logistic model was used to examine overall differences in the number of people who laughed. This was followed by analyses of counts for total laughs between groups. Betweengroup differences in total laughter had to be considered when comparing self-related or other-related laughter, which is most easily done by considering self-related or other-related laughter as a proportion of total laughter. Such proportions do not allow easy distinction between a difference driven by laughing less in one context or more in the other, however, and exclude those who do not laugh at all. We therefore performed two analyses: (1) analysing self-related or other-related laughter as a proportion of total laughter among those who laughed and (2) analysing total counts of self-related or other-related laughter in all participants without adjusting for total laughter. If a group differed in the proportion of laughter in relation to the self or other, but not total counts, we could note the presence of that between-group difference, but not comment on the likely cause. If a group differed in the analysis of laugh counts in relation to self or other, but not proportions, we might suspect a difference in total laughter drove the count difference in relation to self or others. If a group differed on absolute and proportional measures of laughter in relation to self or other, we could comment both on the difference and the probable cause.

For each analysis, a number of potential covariates were analysed to determine whether there were differences among diagnostic groups: (a) total number of syllables spoken by the participant and their partner, (b) disease severity as measured by the Mini-Mental State Exam (MMSE), (c) age and (d) gender. Differences between groups were assessed, and a preselected $\mathrm{p}$ value threshold of $<0.10$ was used to determine inclusion as a covariate.

In the subgroup of laughers included in the analysis of proportions, there were no between-group differences in MMSE score $(\mathrm{p}=0.651$ excluding healthy controls) or in age $(\mathrm{p}=0.17)$. There was a borderline between-group difference in age $(p=0.052)$, and a significant between-group difference in the amount of speech of both the participant and the partner $(\mathrm{p}<0.001)$. When analysing self-related or other-related laughter as a proportion of total laughter, we therefore included age as a covariate. We also included the amount of the partner's speech and laughter in analyses of laughter in relation to others' vocalisations and the amount of the participant's speech in analyses of laughter in relation to one's own vocalisations as covariates.

When not adjusting for total laughter, thereby allowing for inclusion of all participants, analyses revealed no difference in MMSE scores $(p=0.126$ excluding healthy controls). There were significant group differences in gender $(p=0.001)$ and borderline between-group differences for age $(p=0.054)$. We therefore entered both gender and age as covariates in this analysis. As there was again a significant between-group difference in the amount of speech of both the participant and the partner $(p<0.001)$, we adjusted for the amount of the partner's speech and laughter in analyses of laughter in relation to others' vocalisations and the amount of the participant's speech in analyses of laughter in relation to one's own vocalisations. Interactions were assessed and included in all models when present.

In selecting the most appropriate statistical test for each analysis, we considered three potential violations of standard Poisson distributions: non-independence between speakers, overdispersion and zero inflation. Mixed-effects models would be best suited to non-independent data, negative binomial regression to overdispersed data and zero-inflated models for zero inflation. For each of our count-related questions, we used corrected Akaike's information criteria (AIC) and Bayesian information criteria (BIC) to compare Poisson regression and negative binomial regression as well as mixed-effects and zero-inflated versions of each. ${ }^{25-27}$ AIC and BIC indicated Poisson regression as the best model for proportional analysis (ie, when adjusting for total laughter) and negative binomial regression, correcting for overdispersion but not zero inflation or mixed effects, as the best model to compare raw laugh counts within different contexts (see online supplementary table 1).

\section{RESULTS}

\section{Participant demographics}

Demographics for all participants are listed in table 1. Demographics of those who laughed, and thus were included in the

\begin{tabular}{|c|c|c|c|c|c|c|}
\hline & Controls (156) & $\begin{array}{l}A D \\
\text { (17) }\end{array}$ & bvFTD (39) & nfvPPA (16) & svPPA (19) & $\begin{array}{l}\text { rtFTD } \\
\text { (14) }\end{array}$ \\
\hline$\%$ female* & 62.8 & 41.2 & 38.5 & 50.0 & 21.0 & 42.8 \\
\hline$\% \mathrm{R}$ handed & NA & 82.4 & 92.1 & 100.0 & 94.7 & 84.0 \\
\hline Age & $60.4 \pm 10.5$ & $61.2 \pm 7.8$ & $61.4 \pm 8.5$ & $67.6 \pm 10.5$ & $63.7 \pm 7.1$ & $65.5 \pm 5.7$ \\
\hline Education & NA & $16.1 \pm 3.1$ & $16.0 \pm 3.2$ & $17.9 \pm 3.0$ & $17.4 \pm 2.5$ & $16.6 \pm 3.3$ \\
\hline MMSE & NA & $21.0 \pm 5.2$ & $24.6 \pm 4.9$ & $24.8 \pm 8.5$ & $23.9 \pm 3.9$ & $25.7 \pm 3.8$ \\
\hline$\%$ laughers & 69.2 & 64.71 & 56.4 & 75.0 & 73.6 & 57.4 \\
\hline Syllable count * & $849.3 \pm 352.8$ & $539.9 \pm 223.3$ & $417.1 \pm 285.9$ & $445.3 \pm 285.2$ & $873.0 \pm 574.3$ & $574.7 \pm 337$. \\
\hline
\end{tabular}

AD, Alzheimer's disease; bvFTD, behavioural variant frontotemporal dementia; MMSE, Mini-Mental State Exam; nfvPPA, non-fluent variant primary progressive aphasia; rtFTD, right temporal variant frontotemporal dementia; svPPA, semantic variant of primary progressive aphasia. *The asterisk signifies a between-group difference with $p<0.05$. 
Table 2 Demographics for all diagnostic groups, only including those who laughed

\begin{tabular}{|c|c|c|c|c|c|c|}
\hline & Controls (91) & $\begin{array}{l}A D \\
(11)\end{array}$ & bvFTD (22) & nfvPPA (12) & svPPA (14) & $\begin{array}{l}\text { rtFTD } \\
(8)\end{array}$ \\
\hline$\%$ female* & 68.5 & 54.8 & 40.9 & 50.0 & 28.6 & 62.5 \\
\hline$\%$ R handed & NA & 90.9 & 90.9 & 100.00 & 94.7 & 92.9 \\
\hline Age & $60.9 \pm 9.9$ & $58.8 \pm 5.3$ & $60.8 \pm 5.1$ & $66.7 \pm 11.5$ & $65.6 \pm 6.7$ & $66.7 \pm 3.0$ \\
\hline Education & NA & $16.2 \pm 3.0$ & $16.3 \pm 3.6$ & $16.8 \pm 1.8$ & $16.8 \pm 2.4$ & $16.7 \pm 2.9$ \\
\hline MMSE & NA & $22.5 \pm 4.8$ & $24.6 \pm 5.1$ & $24.3 \pm 9.8$ & $23.3 \pm 4.2$ & $26.6 \pm 2.6$ \\
\hline Syllable count * & $840.0 \pm 356.5$ & $496.5 \pm 215.9$ & $472.3 \pm 320.8$ & $476.2 \pm 298.0$ & $699.3 \pm 324.9$ & $734.1 \pm 334.7$ \\
\hline
\end{tabular}

AD, Alzheimer's disease; bvFTD, behavioural variant frontotemporal dementia; MMSE, Mini-Mental State Exam; nfvPPA, non-fluent variant primary progressive aphasia; rtFTD, right temporal variant frontotemporal dementia; svPPA, semantic variant of primary progressive aphasia. The asterisk signifies a between-group difference with $p<0.05$.

analysis of self-related and other-related laughter as a proportion of total laughter, are listed in table 2.

\section{Total laughter and diagnosis}

A total of 1040 laughs were identified in the recordings of the conversations. Non-independence between participants and partners in laughing at least once in the conversation was confirmed at the conversation level $(\operatorname{coef}=2.25, \mathrm{p}<0.001$, CI (1.3 to 4.0$)$ ). A mixed effects logistic regression with a random intercept for each conversation revealed no betweengroup difference in the proportion of people who laughed at least once during the conversation ( $p>0.20$ for all groups). A negative binomial regression of total laugh counts suggested controls laughed more often than those with eoAD (coef -1.56 , $\mathrm{p}=0.007$, CI $(-2.7$ to 0.43$)$ ), $\operatorname{rtFTD}(\operatorname{coef}=-2.43, \mathrm{p}=0.002$, CI $(-3.95$ to -0.91$)$ ) and bvFTD (coef $=-0.97, p=0.009$, CI $(-1.69$ to -0.24$))$. The differences remained after removing potential outliers (figure 1).

\section{Laughter contexts and diagnosis}

We hypothesised that those with bvFTD would laugh less in relation to their own speech than controls. Due to between-group differences in total laughter, we analysed self-related laughter as a proportion of total laughter. This analysis indicated that the proportion of laughter in relation to self versus other was higher in controls than either nfvPPA (coef $=-0.72, \mathrm{p}<0.001$, CI $(-0.95$ to -0.26$)$ ), bvFTD (coef $-0.46, \quad \mathrm{p}=0.001, \mathrm{CI}$ $(-0.74$ to -0.18$)$ ) and svPPA (coef $-0.46, p=0.021$, CI $(-0.88$ to -0.05$)$ ), with a trend for $\operatorname{rtFTD}(\operatorname{coef}=-0.49$, $\mathrm{p}=0.099$, CI (-1.07 to 0.09)) (figure 2).

As noted earlier, treatinwg self-related or other-related laughter as a proportion of total laughter means that results can be driven by more laughter in one context, less in another or some combination of the two. To help clarify the nature of our findings, we also examined the absolute counts of self-related and other-related laughter without considering total laughter. Negative binomial regression indicated more laughter in relation to the self in controls than in eoAD ( $\operatorname{coef}=-0.82, \mathrm{p}=0.049$, CI $(-1.65$ to 0.00$))$, rtFTD $(\operatorname{coef}=-1.19,0.014$, CI $(-2.14$ to $-0.24)$ ) or bvFTD (coef $-0.66, p=0.039$, CI $(-1.3$ to -0.03$)$ ) (figure 3 ). Of these, only bvFTD laughed less in both proportional and absolute terms. While eoAD and rtFTD had less self-related laughter in absolute terms, the lack of any difference in the analysis of proportional data suggests that fewer

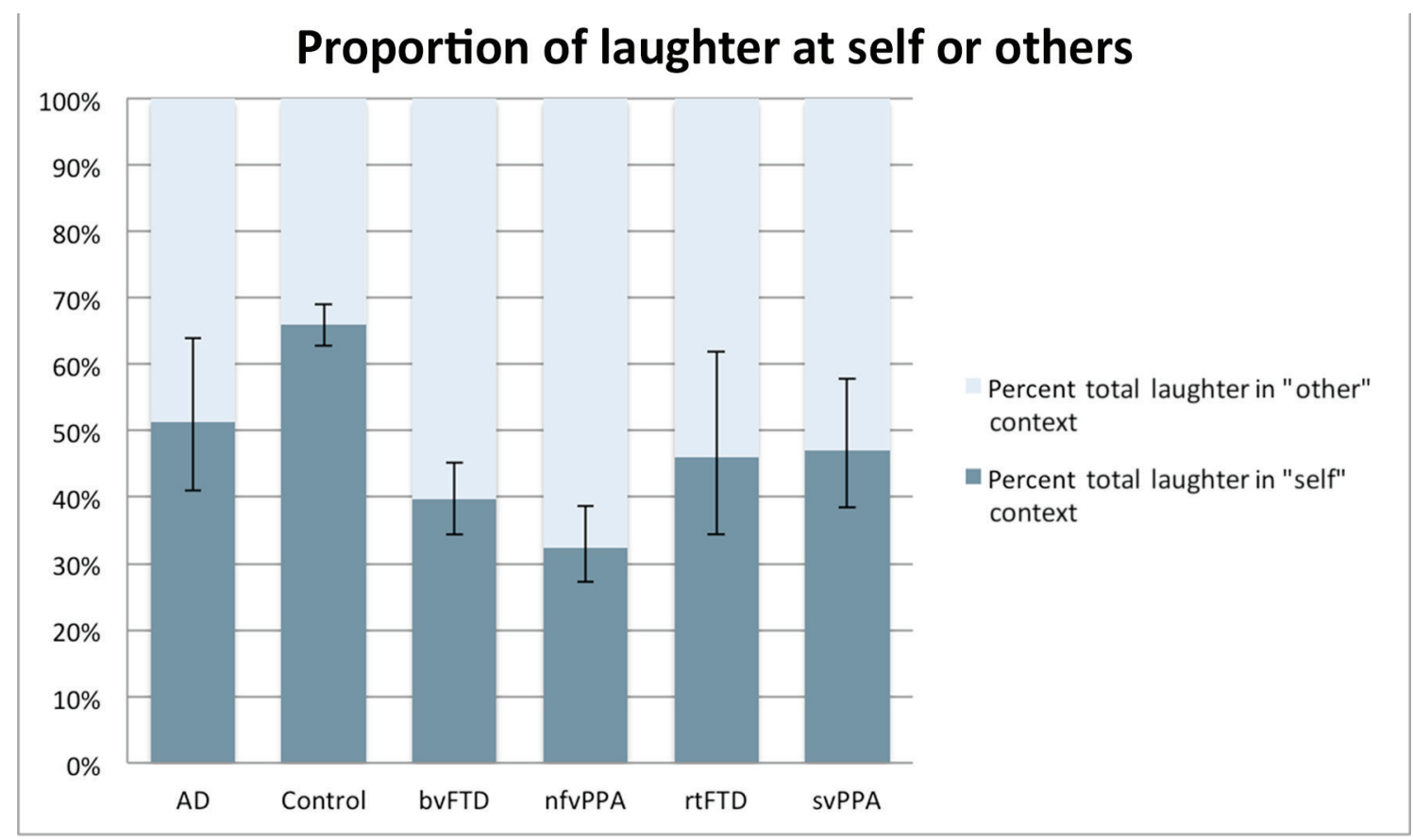

Figure 2 A comparison of laughter in the self or other contexts regarded as a proportion of total laughter. Error bars reflect standard error. Only bvFTD, nfvPPA and svPPA differ significantly from controls. AD, Alzheimer's disease; bvFTD, behavioural variant frontotemporal dementia; nfvPPA, non-fluent variant primary progressive aphasia; rtFTD, right temporal variant frontotemporal dementia; svPPA, semantic variant of primary progressive aphasia. 


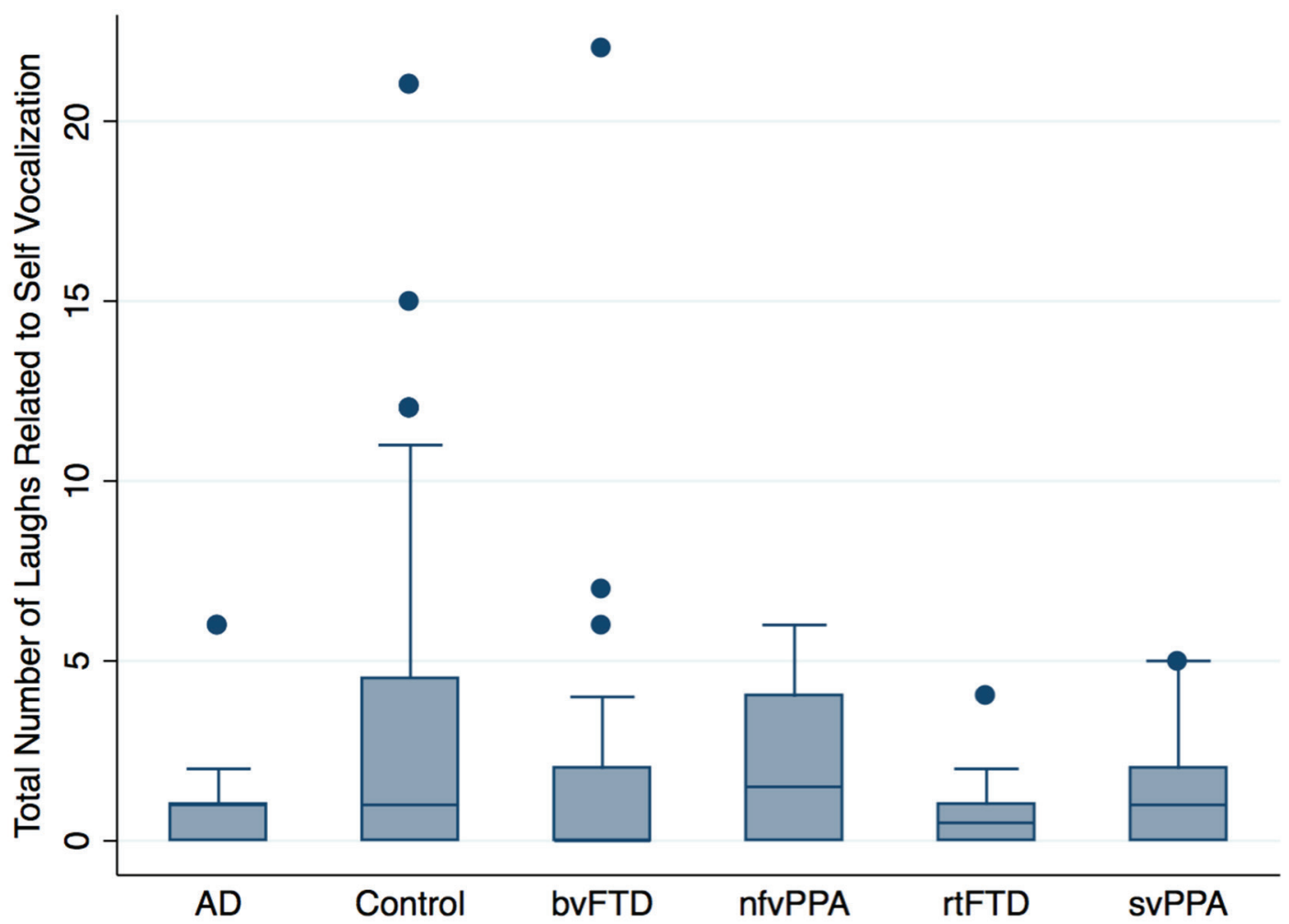

Figure 3 A comparison between different diagnostic groups in the number of conversational laughs during or $<3 \mathrm{~s}$ after one's own speech. On the average, participants with AD, bvFTD and rtFTD laughed less than healthy controls in relation to their own vocalisation. Note, however, that these three also laughed less generally, and of these three only bvFTD differed in the proportion of laughter spent laughing in relation to the self versus others. AD, Alzheimer's disease; bvFTD, behavioural variant frontotemporal dementia; nfvPPA, non-fluent variant primary progressive aphasia; rtFTD, right temporal variant frontotemporal dementia; SVPPA, semantic variant of primary progressive aphasia.

self-related laughs in these patient groups is due to less laughing generally.

We had hypothesised no difference in laughter between those with nfvPPA and controls. However, negative binomial regression suggested that compared with controls, those with nfvPPA were significantly more likely to laugh in relations to others' vocalisations (coef $=1.06, p=0.021$, CI $(0.17$ to 1.94$)$ ) (figure 4). No other diagnostic groups were more or less likely to laugh in relation to others' vocalisations.

\section{DISCUSSION}

We studied laughter during seminaturalistic discussions of a relationship conflict between patients with eoAD, bvFTD, rtFTD, svPPA or nfvPPA and familial caregivers and control dyads. We analysed the total amount of laughter that occurred as well as breaking this down into laughter that occurred in the context of self (ie, following one's one own vocalisations) and in the context of other (ie, following the other person's vocalisations). We hypothesised that patients with bvFTD would laugh less in relation to their own speech and that patients with rtFTD would laugh less overall compared with controls. We did not expect to find differences in laughter between patients with nfvPPA and left predominant svPPA compared with controls.

Our analyses revealed three main findings:

1. Patients with bvFTD, eoAD and rtFTD had fewer total laughs overall compared with controls, with the largest effect found for rtFTD, in accord with our hypotheses.

2. Patients with bvFTD laughed proportionately less in relation to their own vocalisations compared with controls, in accord with our hypotheses.
3. Patients with nfvPPA laughed more than controls in the other context, which was not hypothesised.

\section{Total laughter}

Although all three patient groups who are generally thought to have profound behavioural disturbances (eoAD, bvFTD and rtFTD) laughed less than controls, the effect was strongest in rtFTD. Diminished rates of laughter in this group across both contexts may result from broader loss of semantic knowledge regarding social norms in $\mathrm{rtFTD}^{28}$

\section{Laughter in relation to self versus other}

In order to correct for differences in total laughter, we first regarded laughter in relation to the self or other as a proportion of total laughter. As hypothesised, we found clear differences from controls in the proportion of self-related laughter to other-related laughter in bvFTD. Contrary to our hypothesis, we also found a lower proportion of self-related laughter to other-related laughter in svPPA and nfvPPA, neither of which is typically associated with profound behavioural changes. In order to better understand what was driving the proportional differences, we then analysed absolute laugh counts in each context without consideration of the total laughter. This follow-up analysis of absolute laugh counts without adjusting for total laughter found no difference between svPPA and controls, suggesting that the proportional difference was driven by a combination of diminished laughs in relation to the self and increased laughs in relation to the other, with neither reaching significance when investigated separately. Clearer differences were found regarding 


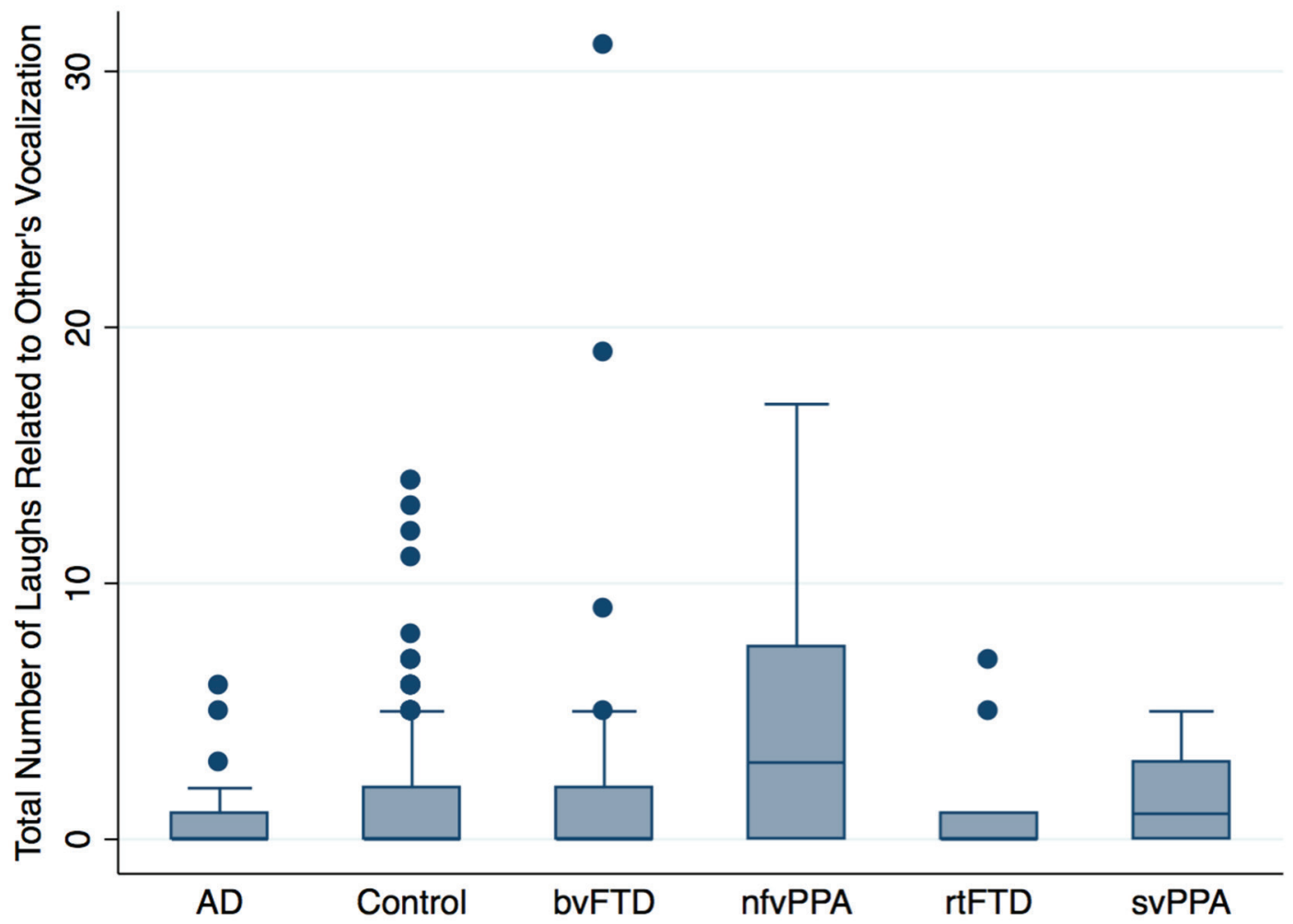

Figure 4 A comparison between different diagnostic groups in the number of conversational laughs during or $<3 \mathrm{~s}$ after another's vocalisations. Those with nfvPPA laugh significantly more than healthy controls in relation to other people's speech. AD, Alzheimer's disease; bvFTD, behavioural variant frontotemporal dementia; nfvPPA, non-fluent variant primary progressive aphasia; rtFTD, right temporal variant frontotemporal dementia; svPPA, semantic variant of primary progressive aphasia.

bvFTD and nfvPPA, with subsequent analyses, unadjusted for total laughter, demonstrating less laughter in relation to self in bvFTD and more laughter in relation to other in nfvPPA.

In combination, the results of our analyses suggest that while those with bvFTD generally laugh significantly less than controls, this reduction seems particularly related to laughter during or shortly after their own utterances. Nervous laughter is part of a healthy self-conscious reaction, and may indicate recognition that one has benignly violated a social norm. Such reactions are clinically diminished in patients with bvFTD, who regularly show little concern at disrupting social mores. Fewer laughs after one's own utterances further support the concept of diminished emotional self-awareness and social self-monitoring in bvFTD. ${ }^{29}$

While nfvPPA was also associated with a decreased self/other proportion in laughter relative to controls, we believe that the reason differed from bvFTDs. Whereas those with bvFTD laughed less in relation to their own vocalisations, patients with nfvPPA laughed more than controls in relation to others. Because nfvPPA is associated with left frontal atrophy, these results may at first appear to contradict studies suggesting that increased jocularity is connected with right, not left, hemispheric lesions. For example, Wada studies that deactivate the right but not the left hemisphere have led to increased laughter, ${ }^{30}{ }^{31}$ and some lesion studies have similarly associated right hemisphere injury with increased laughter. ${ }^{31} 32$ However, these studies did not specifically address whether the laughter was related to self or others. As we have noted, laughter in these contexts involve different kinds of information processing and different associated emotions. Moreover, the relationship between a happiness behaviour and frontal atrophy is likely more complex than these early studies suggest. In a recent study of positive emotional behaviours among patients with FTD (including patients with nfvPPA), greater positive emotion was associated with neurodegeneration in left anterior insula and left ventrolateral prefrontal cortex, both areas atrophied in nfvPPA. ${ }^{33}$ Because the ventrolateral prefrontal cortex is generally associated with behavioural inhibition $^{34}$ and the anterior insula is associated with expressive suppression and behavioural inhibition, ${ }^{35-37}$ degeneration in these areas may serve to disinhibit the expression of positive emotion.

\section{Strengths and limitations}

The purpose of this study was to determine whether patterns of naturally occurring conversational laughter distinguish among different types of FTD and controls. Strengths of this approach included the high ecological validity of using naturalistic conversations between patients with dementia and familial caregivers, objectively measuring laughter, and careful machine-assisted classification of laughter context (self vs others). Limitations include small sample sizes-however, our sample sizes are comparable to other studies of such uncommon neurodegenerative disorders. Other limitations include the lack of more detailed analysis of the particular qualities of the conversation when a laugh occurred, and the lack of anatomical data to test presumed relations between neurodegeneration and laughter.

\section{Future directions}

Finding differences in laughing behaviours between FTD subtypes is encouraging for using this kind of measure 
diagnostically. While the dispersion of laughter behaviour was wide within each diagnostic category, limiting the diagnostic potential of one measurement in time, the diagnostic potential of longitudinal changes in laugh behaviour may merit further study. Future studies should also consider how spontaneous laughter relates to other measures of emotional functioning as well as to anatomical data derived from neuroimaging.

\section{Conclusion}

In a naturalistic social interaction, patients with various forms of FTD, particularly bvFTD, rtFTD and nfvPPA, had different patterns of spontaneous laughter. Future studies should consider the utility of measuring laughter for diagnosing different kinds of dementia, measuring disease severity and tracking disease progression.

Acknowledgements We would like to thank Michael 'Doc' Edge and Dr. Mark Segal for statistical guidance, the patients and staff at the UCSF Memory and Aging Center, as well as research assistants at the Berkeley Psychophysiology Laboratory for coding (Collin Barlow, Abbey Dykhouse, Juliet Small Ernst, Pooja Mahtre, William Harkin, Michelle Hough, Isabel Sible, Christine Silver, Tu Tran and Lily Vittayarukskul).

(c) Article author(s) (or their employer(s) unless otherwise stated in the text of the article) 2017. All rights reserved. No commercial use is permitted unless otherwise expressly granted.

\section{References}

1 Provine RR. Laughing, Tickling, and the Evolution of Speech and Self. Curr Dir Psychol Sci 2004;13:215-8.

2 McGraw AP, Warren C. Benign violations: making immoral behavior funny. Psychol Sci 2010;21:1141-9.

3 Feng S, Ye X, Mao L, et al. The activation of theory of mind network differentiates between point-to-self and point-to-other verbal jokes: an fMRI study. Neurosci Lett 2014:564:32-6.

4 Clark CN, Nicholas JM, Gordon E, et al. Altered sense of humor in dementia. $J$ Alzheimers Dis 2016;49:111-9.

5 Clark CN, Nicholas JM, Henley SM, et al. Humour processing in frontotemporal lobar degeneration: a behavioural and neuroanatomical analysis. Cortex 2015;69:47-59.

6 Neary D, Snowden JS, Gustafson L, et al. Frontotemporal lobar degeneration: a consensus on clinical diagnostic criteria. Neurology 1998;51:1546-54.

7 Pressman PS, Miller BL. Diagnosis and management of behavioral variant frontotemporal dementia. Biol Psychiatry 2014;75:574-81.

8 Rascovsky K, Grossman M. Clinical diagnostic criteria and classification controversies in frontotemporal lobar degeneration. Int Rev Psychiatry 2013:25:145-58.

9 Sturm VE, Ascher EA, Miller BL, et al. Diminished self-conscious emotional responding in frontotemporal lobar degeneration patients. Emotion 2008;8:861-9.

10 Kamminga J, Kumfor F, Burrell JR, et al. Differentiating between right-lateralised semantic dementia and behavioural-variant frontotemporal dementia: an examination of clinical characteristics and emotion processing. J Neurol Neurosurg Psychiatry 2015;86:1082-8.

11 Seeley WW, Bauer AM, Miller BL, et al. The natural history of temporal variant frontotemporal dementia. Neurology 2005;64:1384-90.

12 Van Langenhove T, Leyton CE, Piguet 0 , et al. Comparing longitudinal behavior changes in the primary progressive aphasias. J Alzheimers Dis 2016;53:1033-42.

13 Mandelli ML, Vitali P, Santos M, et al. Two insular regions are differentially involved in behavioral variant FTD and nonfluent/agrammatic variant PPA. Cortex 2016;74:149-57
14 Ossenkoppele R, Pijnenburg YA, Perry DC, et al. The behavioural/dysexecutive variant of Alzheimer's disease: clinical, neuroimaging and pathological features. Brain 2015; 138:2732-49.

15 Goodkind MS, Sturm VE, Ascher EA, et al. Emotion recognition in frontotemporal dementia and Alzheimer's disease: a new film-based assessment. Emotion 2015;15:416-27.

16 McGraw AP, Warren C. Benign violations: making immoral behavior funny. Psychol Sci 2010;21:1141-9.

17 McGraw AP, Warren C, Williams LE, et al. Too close for comfort, or too far to care? Finding humor in distant tragedies and close mishaps. Psychol Sci 2012;23:1215-23.

18 Neary D, Snowden JS, Gustafson L, et al. Frontotemporal lobar degeneration: a consensus on clinical diagnostic criteria. Neurology 1998;51:1546-54.

19 Provine R. Laughter punctuates speech: linguistic, social and gender contexts of laughter. Ethology 1993;95:291-8.

20 Khachaturian ZS. Revised criteria for diagnosis of Alzheimer's disease: National Institute on Aging-Alzheimer's Association diagnostic guidelines for Alzheimer's disease. Alzheimers Dement 2011;7:253-6.

21 Kamminga J, Kumfor F, Burrell JR, et al. Differentiating between right-lateralised semantic dementia and behavioural-variant frontotemporal dementia: an examination of clinical characteristics and emotion processing. J Neurol Neurosurg Psychiatry 2015;86:1082-8.

22 Levenson RW, Gottman JM. Marital interaction: physiological linkage and affective exchange. J Pers Soc Psychol 1983;45:587-97.

23 Boersma P. Praat, a system for doing phonetics by computer. Glot International 2001;5:341-5.

24 Provine R. Laughter punctuates speech: linguistic, social and gender contexts of laughter. Ethology 1993;95:291-8.

25 Akaike H. A new look at the statistical model identification. IEEE Trans Automat Contr 1974;19:716-23.

26 Burnham K, Anderson D. Model selection and multimodel inference: a practical information-theoretic approach. 2 ed. New York: Springer, 2002.

27 Schwarz G. Estimating the dimension of a model. The Annals of Statistics 1978:6:461-4.

28 Pobric G, Lambon Ralph MA, Zahn R. Hemispheric specialization within the superior anterior temporal cortex for social and nonsocial concepts. J Cogn Neurosci 2016;28:351-60.

29 Weisfeld GE, Weisfeld MB. Does a humorous element characterize embarrassment? Humor-International journal of humor research. 2014:27:65-85.

30 Perria L, Rosadini G, Rossi GF. Determination of side of cerebral dominance with amobarbital. Arch Neurol 1961;4:173-81.

31 Sackeim HA, Greenberg MS, Weiman AL, et al. Hemispheric asymmetry in the expression of positive and negative emotions. neurologic evidence. Arch Neurol 1982:39:210-8

32 Gainotti G. Emotional behavior and hemispheric side of the lesion. Cortex 1972;8:41-55.

33 Sturm VE, Yokoyama JS, Eckart JA, et al. Damage to left frontal regulatory circuits produces greater positive emotional reactivity in frontotemporal dementia. Cortex 2015;64:55-67.

34 Aron AR. The neural basis of inhibition in cognitive control. Neuroscientist 2007; 13:214-28

35 Giuliani NR, Drabant EM, Bhatnagar R, et al. Emotion regulation and brain plasticity: expressive suppression use predicts anterior insula volume. Neuroimage 2011;58:10-15.

36 Jezzini A, Caruana F, Stoianov I, et al. Functional organization of the insula and inner perisylvian regions. Proc Natl Acad Sci U S A 2012;109:10077-82.

37 Kurth F, Zilles K, Fox PT, et al. A link between the systems: functional differentiation and integration within the human insula revealed by meta-analysis. Brain Struct Funct 2010;214:519-34.

38 R: a language and environment for statistical computing. Vienna, Austria: R Foundation for Statistical Computing, 2014. [program]. 3.1.2version.

39 Stata Statistical Software: Release 13 [program]. College Station, TX: StataCorp LP, 2013.

40 Praat [program]. 5.4.01 version. 2014. 